\title{
The effects of parecoxib on movement-related pain following major gynecological surgery
}

\author{
M.N. Essex, ${ }^{1}$ R. Cheung, ${ }^{1}$ C. Li ${ }^{1}$ \\ ${ }^{1}$ Pfizer Inc, New York, NY, USA
}

\section{INTRODUCTION}

- Early mobilization is a component of enhanced recovery after surgery protocols and helps improve outcomes following surgery, although pain is a major deterrent to early mobilzation. ${ }^{1}$

- Opioids are common in the postoperative setting, although current guidelines recommend a multimodal analgesic approach in which patients receive an around-the-clock regimen of nonselective nonsteroidal anti-inflammatory drugs (NSAIDs), cyclooxygenase-2 selective NSAIDs (coxibs), and/or acetaminophen unless contraindicated. ${ }^{2}$

- Opioids are effective for pain at rest but less effective for movement-induced pain. ${ }^{3}$ Nonselective NSAIDs are effective for movement-induced pain but may result in a variety of side effects, including gastrointestinal (GI) ulceration and bleeding. ${ }^{4,5}$

- Coxibs can provide analgesia with less risk of bleeding and GI events. ${ }^{6}$

- Parecoxib is an injectable coxib approved in over 80 countries for the management of postoperative pain, although few studies have specifically examined parecoxib for movement-induced pain.

- The current study examined the efficacy of parecoxib for movementrelated pain following major gynecological surgery.

\section{METHODS}

- Data were pooled from 2 randomized trials in which patients received a single IV injection of placebo $(n=87)$, morphine 4 $\mathrm{mg}(\mathrm{n}=82)$, ketorolac $30 \mathrm{mg}(\mathrm{n}=83)$, parecoxib $20 \mathrm{mg}(\mathrm{n}=79)$, or parecoxib $40 \mathrm{mg}(\mathrm{n}=79)$ the day after major gynecological surgery. ${ }^{4,7}$ - Pain intensity and pain relief were assessed regularly from 0.25 to $24 \mathrm{~h}$ postdosing, whereas evaluation of study medication was assessed at $24 \mathrm{~h}$. At each time point, patients also answered yes or no to the question "my starting pain is at least half gone."

- Pain was assessed following an aggravating "log-roll" movement in which patients rolled to 1 side, followed by a return to their original position, with the head of the bed at a $30^{\circ}$ incline.

- Treatment effects were compared between groups using analysis of covariance (pain intensity) or analysis of variance (pain relief and study medication scores) models. The time to a $\geq 50 \%$ decrease in pain was calculated using the Kaplan-Meier approach and compared between groups using a log-rank test.

\section{RESULTS}

- Patient demographics were similar between treatment groups (data not shown).

- Mean pain intensity scores were similar among all treatment groups at $0.25 \mathrm{~h}$ postdose (Figure 1).

- All active treatments exhibited significantly lower pain intensity scores relative to placebo from 0.5 to $1.5 \mathrm{~h}$ with the exception of parecoxib $20 \mathrm{mg}$ at $0.5 \mathrm{~h}$ (all $\mathrm{p}<0.05$ ).

- In contrast to morphine, the ketorolac and parecoxib groups exhibited sustained reductions in pain intensity relative to placebo at 2 to $24 \mathrm{~h}$ (all $\mathrm{p}<0.05)$.

\section{RESULTS (cont'd)}

Figure 1. Change in mean pain intensity scores over time. Scores range from $0=$ no pain to $100=$ worst pain.

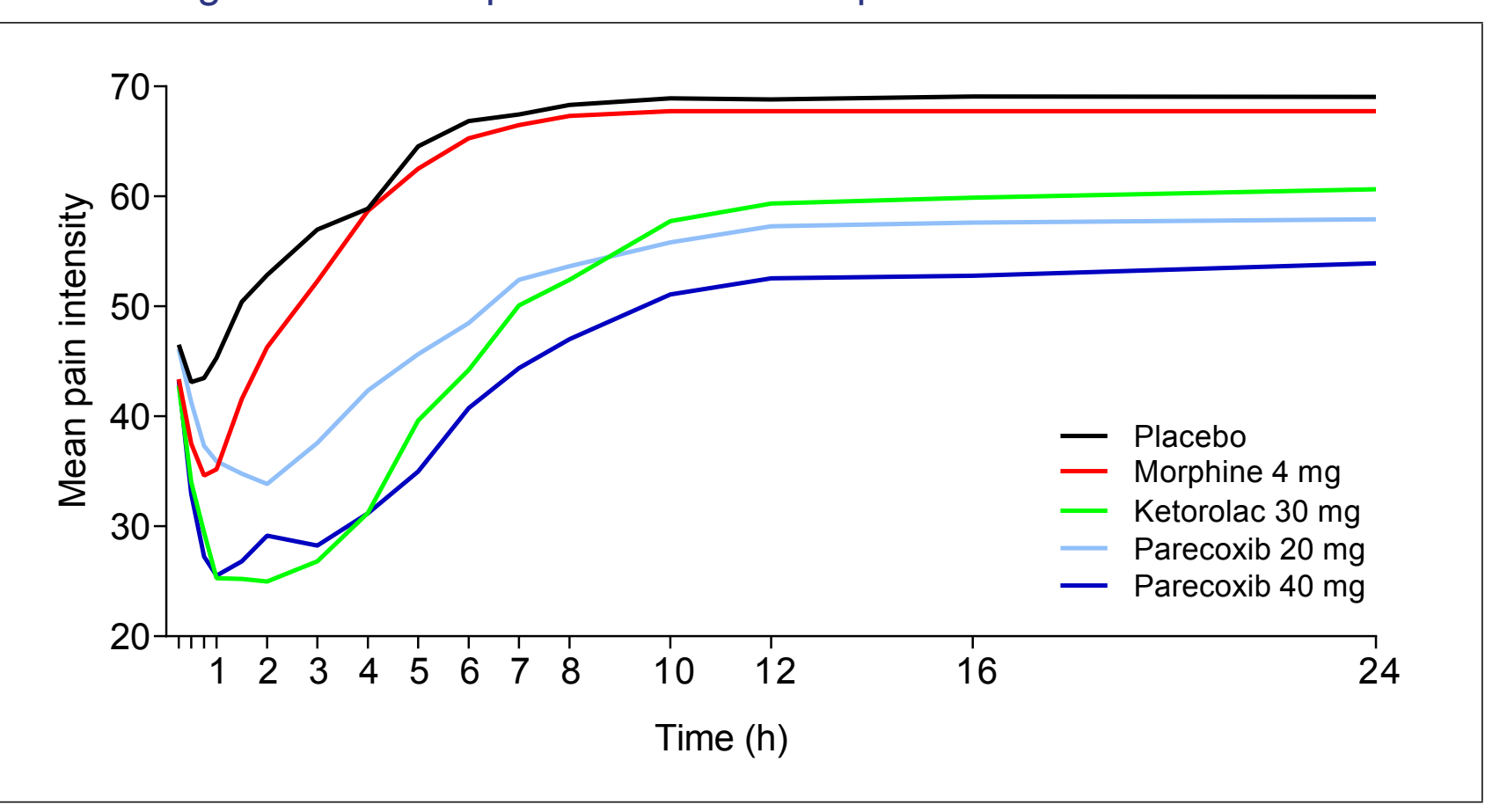

- Mean pain relief scores were significantly higher for all treatment groups relative to placebo at 0.25 to $1.5 \mathrm{~h}$, with the exception of parecoxib $20 \mathrm{mg}$ at $0.25 \mathrm{~h}$ (all $\mathrm{p}<0.05$; Figure 2).

- In contrast to morphine, the ketorolac and parecoxib groups exhibited sustained pain relief relative to placebo at 2.0 to $24 \mathrm{~h}$ (all $\mathrm{p}<0.05$ ).

Figure 2. Change in mean pain relief scores over time. Scores range from $0=$ none to $4=$ complete.

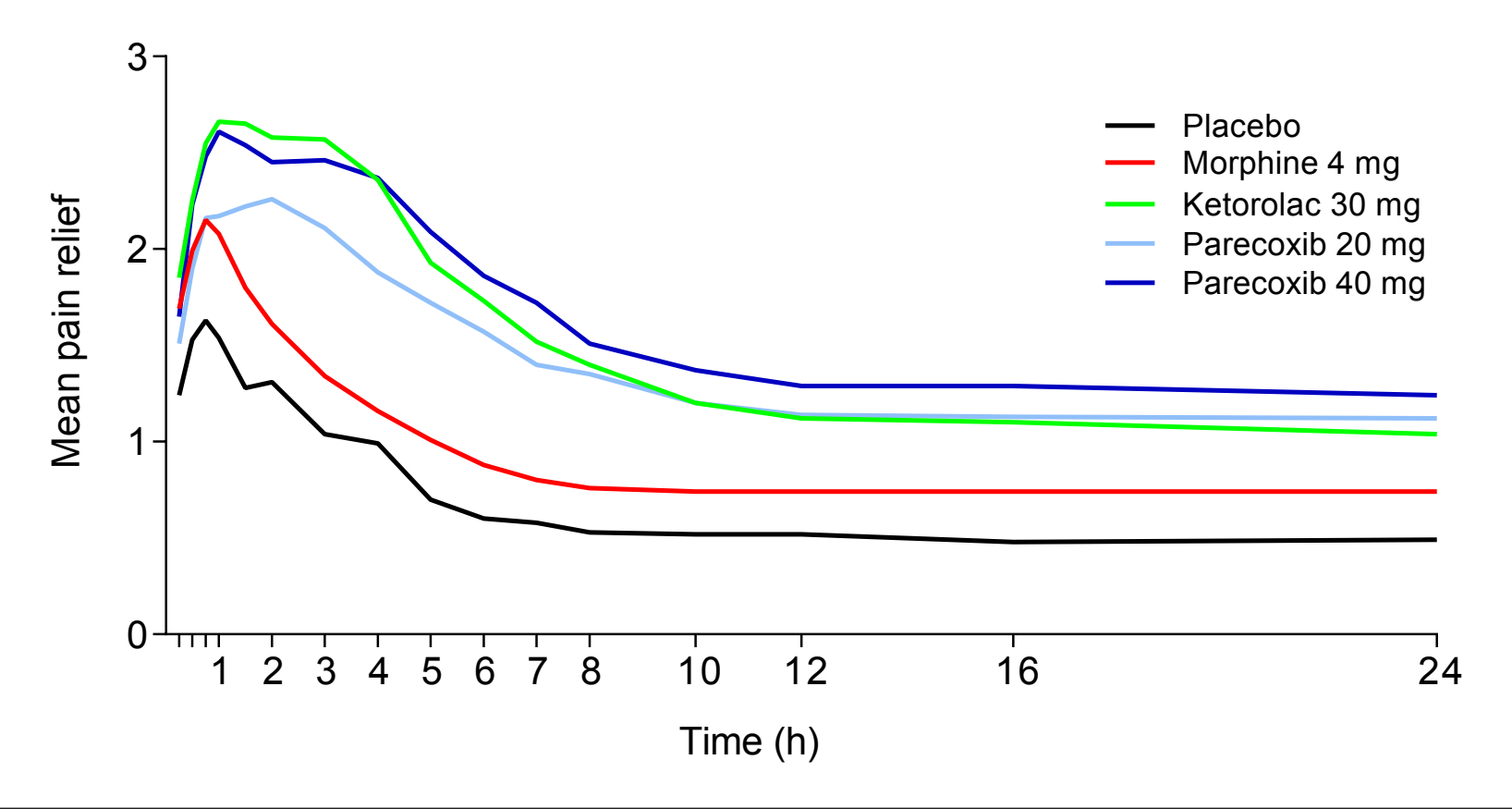

- Median time to $a \geq 50 \%$ reduction in pain was shorter for morphine (23 min), ketorolac (16 min), parecoxib $20 \mathrm{mg}$ (26 min), and parecoxib $40 \mathrm{mg}$ (23 $\mathrm{min}$ ) relative to placebo $(45 \mathrm{~min})($ all $\mathrm{p}<0.05)$.

- Patient evaluation of study medication scores were also better in all treatment groups relative to placebo (all $p<0.05$; data not shown).

- The percentage of patients rating treatment as good or excellent was $51 \%$ for morphine, $78 \%$ for ketorolac, $64 \%$ for parecoxib $20 \mathrm{mg}, 73 \%$ for parecoxib $40 \mathrm{mg}$, and $29 \%$ for placebo.

\section{CONCLUSIONS}

- Although all active treatments reduced movement-induced pain in the $2 \mathrm{~h}$ following major gynecological surgery, the parecoxib 20-mg, parecoxib 40-mg, and ketorolac 30-mg groups provided significantly greater pain relief compared with morphine $4 \mathrm{mg}$ over the full 24-h period.

- In general, the effects of ketorolac $30 \mathrm{mg}$ and parecoxib $40 \mathrm{mg}$ were similar over the full $24-\mathrm{h}$ period. 